Historic, archived document

Do not assume content reflects current scientific knowledge, policies, or practices. 


\section{GRASS SEED}

Timoth", fancy gra le.... $\%$ : 91

Kentcky Bu? Grass, ...... 6.00

Canzilian Blue Grass..... 22.00

Enclish Ry Grass.......... 1400

Italian Rye Grass......... 1400 CHICK AND ANIMAL FOOD

Brome Grass........... 15.no Pease's Pevic Chic z Fod

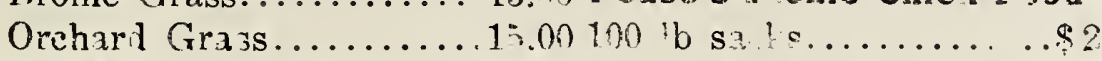

Ped Top.

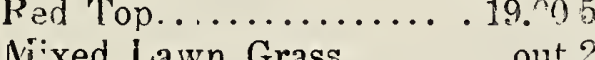

MISOSLLANEOUS

Gull $\ldots \ldots \ldots$..........

Brol:en sack lots or

Ye low Pod Varietie

Golden Wax Pust Proof.

Black Wax Pro fic......... 15

MISCELLANEOUS VINE SEED

Cu umber--White Spine... 55

en weight orde s......... Green Pod Varisties

850 Giant Stringless.

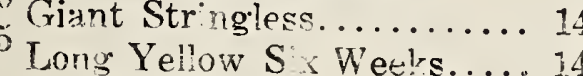

Red Valentine......... j4 Fie rerson Pus in ...... 18

j4: Scurash-Mam.Summer Cr'reck 65 Pumkkin--Small Sugar...... 40 “ Connecticut Field 35 Kentucky Field.... 22 Warted Hubbard.... 65 Hubbard.........6.65 45 brukt Wonier (pic) ...

$18 \mathrm{Msk}$ Melon--Rocky Ford.... 45 Pease's Balanced Chick Chop

CLOVER

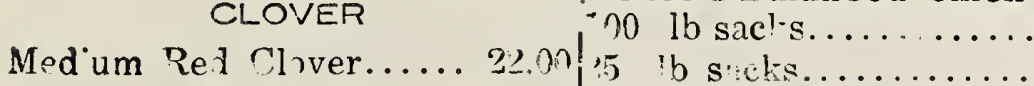

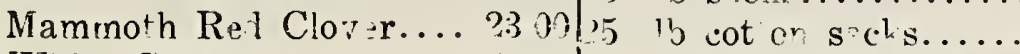

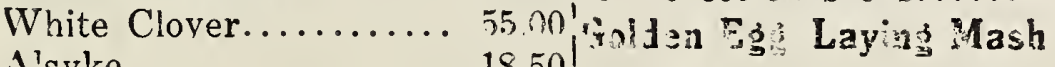

A'syke.................. 1850

Sweet Clover (white bloss $m$ ) Un hulled seed......8. 8.25

Hulled seed........12.00 Bear's Banced hon Chop

\section{(.) jiasta.}

$6 ?$ tro the.ucin Won tre.......

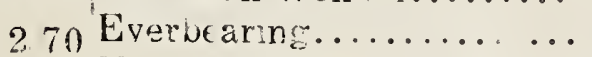
.70 lor: I humb.

SWELT CORN

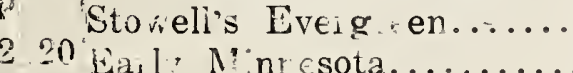

\section{Sto well's Eveig icen.....}

Alfalfa: fancy........ 19.0

Alfalfa, choire........ 17. $n$

Alfalfa, prime........ 13.00

SORGO, KAFFIR \& MILLET

Amber Cane Fodder..........

Orange Cane $\ldots \ldots \ldots \ldots \ldots \ldots \ldots$
Feterita $\ldots \ldots \ldots \ldots \ldots \ldots \ldots$

White Kaffir........... 2.25

CYCLONE SEED SOWERS

Feed Kaffir............. $200 \mathrm{~A}$ hand--sover; adjustab'e for

Golden Liberty Millet.......... zny grain or seed

Siherian Millet............. Sing'e scrers...

White Wcn ler Millet

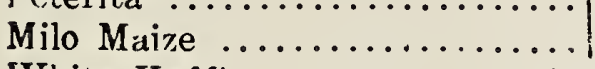

Per Che Lasl: N!ntesola...........

\& 7 . Sountry Gentleman........ 12 4.50 CABBAGE

$3.75 \mathrm{Harly} J \in \mathrm{C}^{2}$ 3.75 Early Flat Dutch........ 1.10

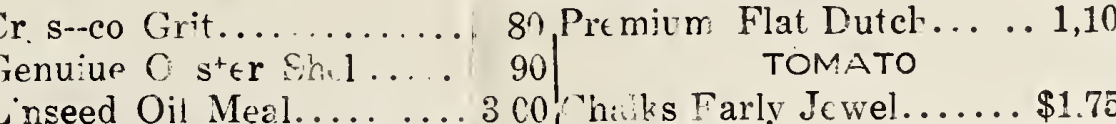

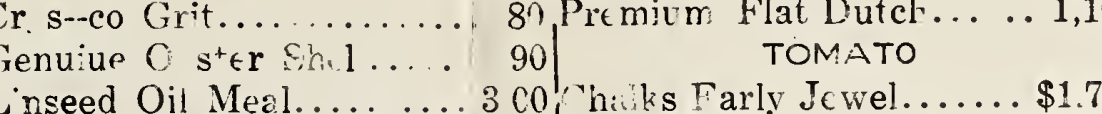
Mat hlcss .......... 1.75 $\begin{aligned} & \text { LETTUCE } \\ & \text { Ein pson:s Earl! Curler..... }\end{aligned}$ \$i.goir arsen's Hearlirg.......... 5.50 Blaek Sefded "impson.
Greer I u neg 60 is " 12) “ Kleckly Sucet 35 $1+$ is “ Gyrsy or Gecr-$1 \div 2$ 34 14) ROOT VEC TAELES Parsnip-Hollow Crcwn.... 35 Carrot--Eaily Oxheart..... 45 c9) "Lanver's half Long 45 Tu nip--White Globe ....... 35 " Pirple Top S 1 r, I.eaf 35 " Am. Yellow Rutabaga 35 Гe ts--Early Blocd Turnip... 35 " Red Mangel W'urtzel .. 23 Rad:sh--White Icicle....... 45 Early Scarlet Turnip 42 Ei riy Iong Scarlet 42 French Breakfast... 42 Onion--W'hite Silver Skin . . 1.40 6) " Red W'eathersfield .. 1,40 65 "Southport Yel. Globe 1.40 60 ONICN SETS MKT.PRICE

We give no warranty exnressed or imp ted, as to despription, quality, procu tivencss or ary other natter of any seed we send out and we will not he in anv way resnonsih! for the crop cown llaref rem. We the purchaser coes not accent the goods on these terms, he must notify us at , nce and we will give instructions for the disgosiston of the gonds.
PEASE GRAN AND SEED CO. 


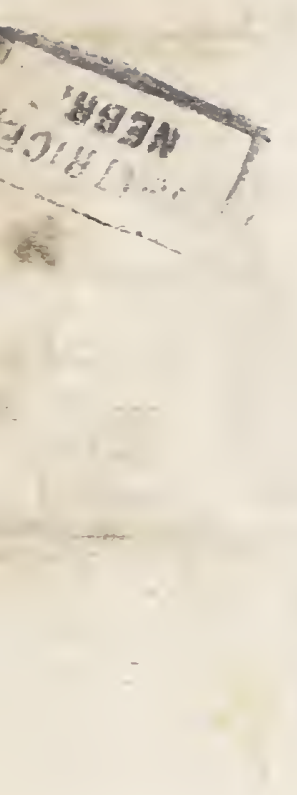

